\title{
Traffic Monitoring System Using IoT and DL
}

\author{
Jayakumar $\mathrm{S}^{\mathrm{a}, 1}$, Lokesh Kumar $\mathrm{K}^{\mathrm{b}}$, Purva Darshini $\mathrm{S} \mathrm{K}^{\mathrm{b}}$ and Sanjeev $\mathrm{D}^{\mathrm{b}}$ \\ a Assistant Professor, Department of Electronics and Instrumentation Engineering, KCG \\ college of technology, Chennai \\ ${ }^{\mathrm{b}}$ Students, Department of Electronics and Instrumentation Engineering, KCG college of \\ technology, Chennai
}

\begin{abstract}
In Metropolitan cities, where the amount of automobile continuously expands faster than the obtainable traffic framework to support them, congestion may be a strenuous issue to affect and it becomes much worse in case of car accidents. This problem affects many aspects of contemporary society, health damages, traffic accidents, duration spent, grow in greenhouse emissions and including economic expansion. In this context, current societies can rely on the traffic management system to diminish traffic congestion and its negative chattels. In this project, we propose a traffic management system where the traffic will be monitored with all the automobiles on the road. We will track the number of automobiles entering the signal zone and will predict the traffic is high or low. Hang on the quantity of automobiles in each signal zone, the traffic signals can be automated. So that maximum amount of duration is given to more automobiles whereas the lesser duration for some automobiles. Thus this project aims at reducing the traffic and managing the signals automatically leading to the sensor less traffic management system.
\end{abstract}

Keywords. Traffic Signal monitoring, recommendation system, Image processing, deep learning.

\section{Introduction}

Traffic congestion in four of Indias major cities Mumbai, Bengaluru, Delhi, and Pune have crawled back very on the brink of levels seen before the Convidinduced lockdown, which had originated global annual traffic congestion to fall for the first duration in at least 10 years, Traffic congestion is a condition in movement that is characterized by low speed, overlong trip duration, and expansion automotive queueing. Traffic congestion on urban road networks has growd substantially, since the 1950s. When traffic demand is great enough that the interaction between automobiles lows the speed of the traffic stream, this leads to some congestion. While congestion may be a possibility for any mode of transportation, this text will specialize in automobile congestion on public roads. As demand approaches the capacity of a road (or of the intersections along the road), extreme traffic jams sets in. When automobiles are fully stopped for periods, this is known as a traffic jam or (informally) a traffic snarl-up. About half of U.S. traffic

\footnotetext{
${ }^{1}$ Jaykumar S, Department of EIE, KCG college of technology, Chennai, India.

E-mail: jayakumar.ei@kcgcollege.com.
} 
congestion is recurring, and is attributed to the sheer weight of traffic; most of the rest is attributed to traffic incidents, road work, and weather events. In terms of traffic operation, rainfall reduces traffic capacity and operating speeds, thereby leading to greater congestion and road net-work productivity loss. Traffic research still cannot fully predict under which conditions a "traffic jam" (as opposed to heavy, but smoothly flowing traffic) may suddenly occur. It has been establish that individual incidents (such as accidents or maybe one car braking heavily during a previously smooth flow) may originate ripple chattels (a cascading failure) which then spread out and create a sustained traffic jam when, otherwise, the usual flow might have recommenced for some duration overlong.

\section{Literature Survey}

The literature review of the paper that were referenced and those that serves the base paper and supporting paper which provides the clear idea of the traffic monitoring system and machine learning algorithms and proper method of recommendations. [1-3] Due to the swift enlargement of automotive transportation and urbanization, traffic jam has been enlarging and becomes a significant problem in most major cities worldwide. Then, an algorithm is presented to spot congested road segments and construct congestion propagation graphs to model congestion propagation in urban road networks $[4,5]$. Traffic jams are also coupled with accidents which can be seen widely [6]. There is also need for rerouting traffic for ambulance and emergency occasion [7]. [8-14] Autonomous and connected cars (ACCs), together with edge computing (EC), have been recognized as a promising solution to realize green intelligent transportation for smart cities. This paper aims to deal with short-term traffic prediction, a fundamental enabler for the success of ACC applications, under the ECU Telecommunications Standards Institute (ETSI) multiaccess EC (MEC) architecture that exhibits different constraints from conventional cloud computing.

\section{Methodology}

The Table 1 Show The hardware Components Used To develop A Device. In This Arduino UNO Is the Microprocessor, Max30100, Ec0567, Ad8232 Are the Sensor That Are Connected In The Human Body. The sensor that are connected in the human body. The LCD used for the display

Table 1. Hardware Components table

\begin{tabular}{|c|l|l|}
\hline S.No & Name of the components & Description \\
\hline 1 & ESP8266 & $\begin{array}{l}\text { Send data to the MySQL Database through } \\
\text { Wi-Fi module. }\end{array}$ \\
\hline 2 & MQ-5 & To sense the atmospheric gas \\
\hline 3 & ATmega-328 & Microcontroller which has access to MQ-5 \\
\hline
\end{tabular}




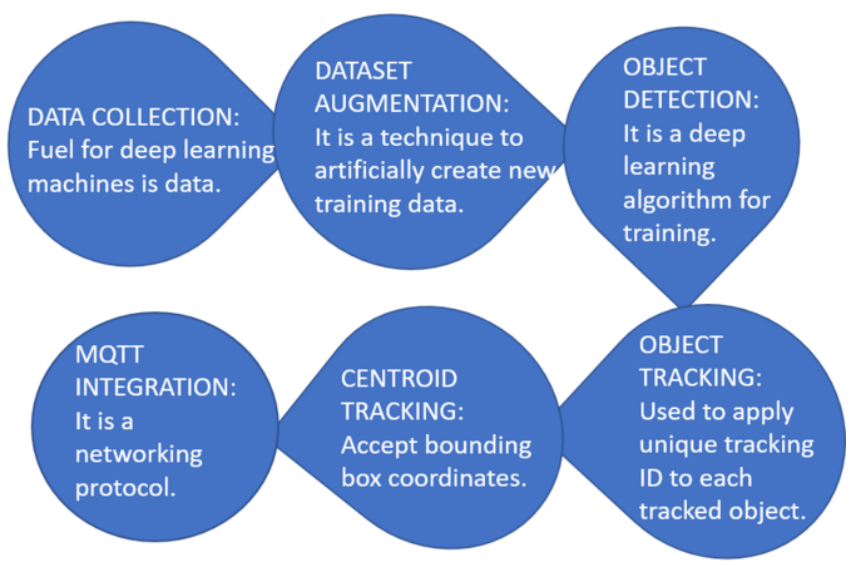

Figure 1. Methodology of the system

\subsection{Hardware parts}

\subsubsection{Esp8266 Module}

The enlargement board consisting of the ESP-12E module is incorporated with the ESP8266 chip having 32-bit LX106 RISC microprocessor. The Board operates at a clock frequency of $80 \mathrm{MHz}$ to $160 \mathrm{MHz}$. Most of the IoT projects commonly use the NodeMCU module which is capable of storing data and programs in its 4MB flash memory. NodeMCU is powered through a micro-USB inlet and has inbuilt Wi-Fi support. It supports UART, SPI, and I2C interface.

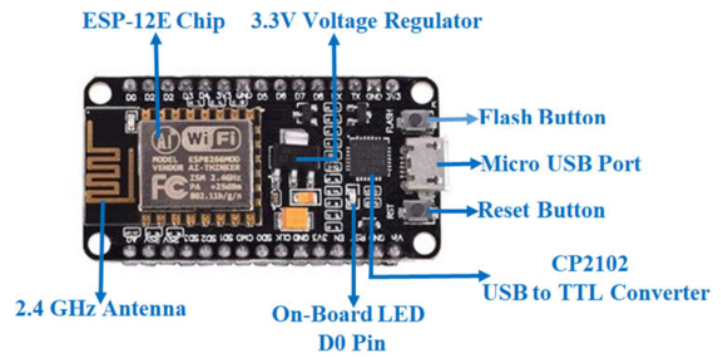

Figure 2. ESP8266

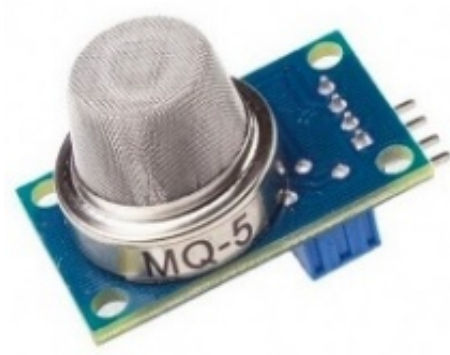

Figure 3. MQ-5

\subsubsection{MQ-5 Gas Sensor}

The resistance of the $\mathrm{SnO} 2$ filament drops, which ends up during a corresponding rise in Output Voltage (Vout), and this output voltage may be measured to point the concentration of any flammable gas that's a gift. The MQ-5 has Associate in Nursing analog (voltage) output. 


\subsubsection{ATmega- 328}

ATmega-328 is a complicated Virtual reduced instruction set computer (AVR) microcontroller. It supports the information up to eight (8) bits. ATmega-328 has 32KB internal integral memory. This micro-controller contains a heap of alternative characteristics. you ought to even have a glance at Introduction to PIC16F877a (it's a PIC Microcontroller) then compare the functions of those 2 Microcontrollers. ATmega 328 has $1 \mathrm{~KB}$ Electrically effaceable Programmable storage (EEPROM). This property shows if the electrical provide equipped to the micro-controller is removed, even then it will store the information and might give results once providing it with the electrical provide.

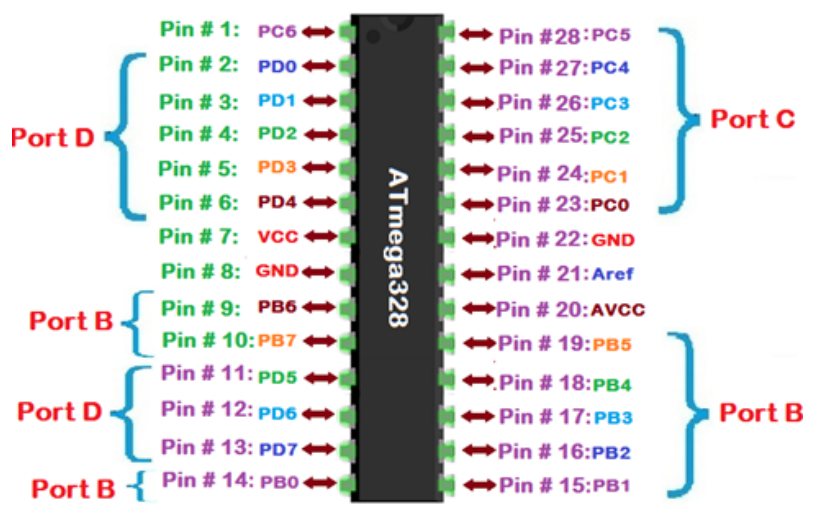

Figure 4. ATmega-328

\subsection{Module Description}

\subsubsection{Dataset Collection Module}

A data set is a collection of data. Machine Learning [15] has become the go-to method for solving many challenging real-world problems. And, in the deep learning era, data is very well arguably the most valuable resource. Mighty has been doing self-driving car image annotation and has become pretty big in the space where CVPR 2018 too. Payment $\mathrm{AI}$ is less specialized than Mighty AI, offering image annotation for any domain.

\subsubsection{Dataset Augmentation}

Dataset augmentation is a method in which a new training data is derived from the existing data. Domain specific techniques are applied to the examples from the training data that results in creating a new and different training examples. Image data augmentation is usually only applied to the training dataset, and to not the validation or test dataset. This is different from data preparation like image resizing and pixel scaling; they need to be performed consistently across all datasets that interact with the model

\subsubsection{Object Detection Training}

Single Shot Multi-Box Detector could be a fashionable algorithmic rule in object detection. After trying an exact of convolutions for feature extraction, we've got a bent to 


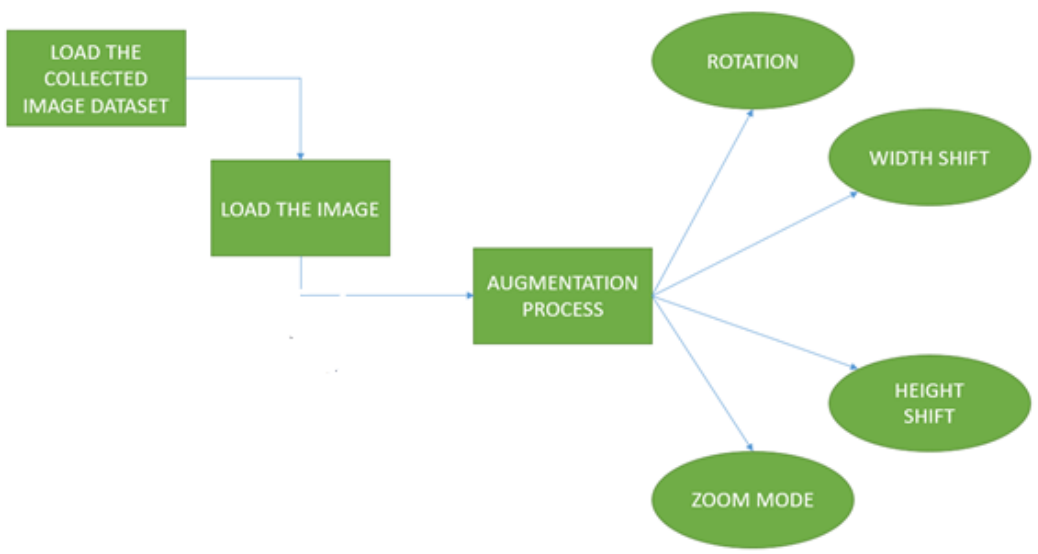

Figure 5. Data augmentation

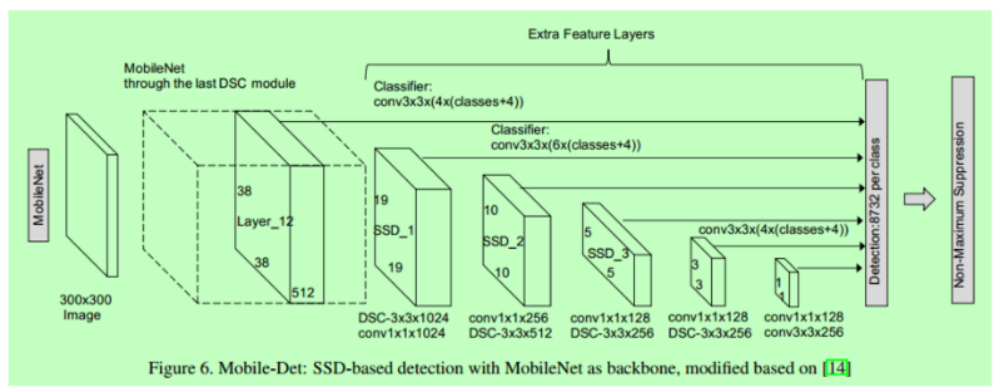

Figure 6. Mobile net SSD architecture

induce a feature layer of size mn (number of locations) with p channels, like 88 or 44 more than. And a 33 convolution is applied on this mnp feature layer. For each location, we've got a bent to got $\mathrm{k}$ bounding boxes. These $\mathrm{k}$ bounding boxes have whole different sizes and aspect ratios.

\subsubsection{Object Tracking}

Object chase has many smart applications similarly as investigating, medical imaging, traffic flow analysis, self-driving cars, people investigation and audience flow analysis, and human-computer interaction. Technically, object chase starts with object detection identifying objects in a very image and distribution them bounding boxes. the article chase algorithm assigns Associate in Nursing ID to each object illustrious at intervals the image, and in resultant frames tries to carry across this ID and establish the new position of a constant object. Their unit two main kinds of object tracking: Offline object chaseobject trailing on a recorded video where all the frames, similarly as future activity, unit well-known ahead. 


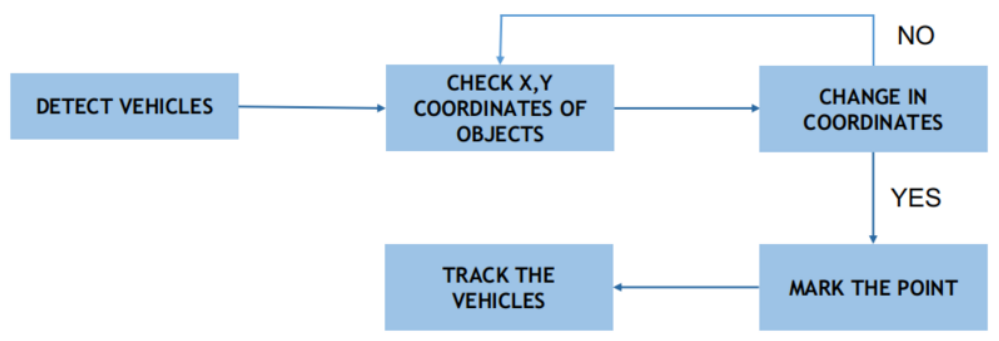

Figure 7. Object Tracking
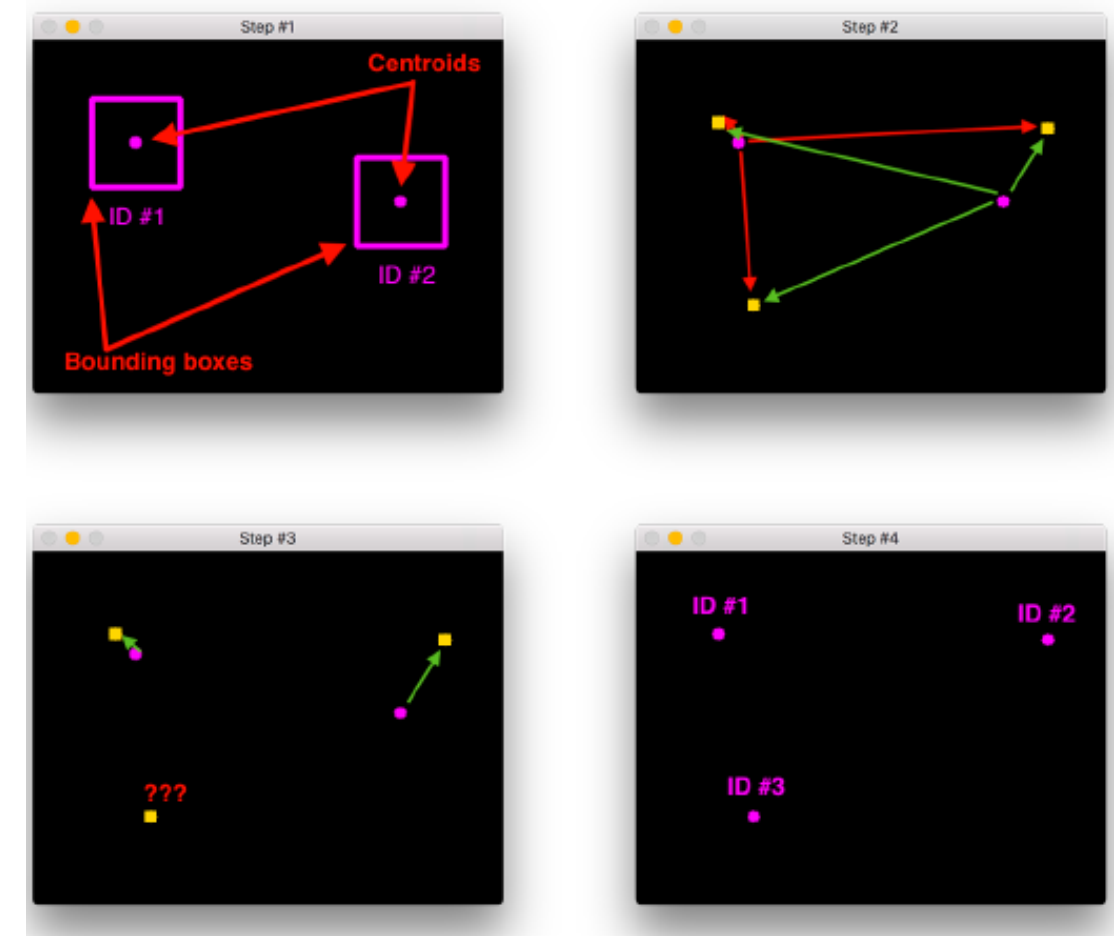

Figure 8. Centroid tracking

\subsubsection{Centroid Tracking}

Update (x, y)-coordinates of existing objects Register new object - Registering just suggests that we've got a bent to unit adding the new object to our list of caterpillar-tracked objects by (1) distribution it a replacement object ID, and (2) storing the center of mass of the bounding box coordinates for that object Deregister recent/lost objects that have removed of the frame - we have a tendency to square measure reaching to deregister recent objects once they cannot be matched to any existing objects for a whole of $\mathrm{N}$ succeeding frame. Here we wish to assign the direction mode, either "vertical" or "horizontal", that we tend to area unit about to be reckoning our objects. The parameters just like the peak and dimension of the input image given. If the direction mode is "vertical", exclusively the popular and total down. Similarly, if direction mode is "horizontal, exclusively the 
popular right and total left thought of. This module accepts a traceable object And one center of mass.

\subsubsection{MQTT Integration}

The protocol popularly runs over TCP/IP but any network protocol that gives ordered, lossless, bi-directional connections can support MQTT. It's designed for connections with remote locations where a "small code footprint" is required or the network system of measurement is out. Associate in Nursing MQTT client is Associate in Nursing device that runs AN MQTT library Associate in Nursing links to Associate in Nursing MQTT broker upon network knowledge is organized in an extremely hierarchy of topics. This might be given by the basic protocol transport exploitation measures to safeguard the unity of transferred knowledge from interception or duplication

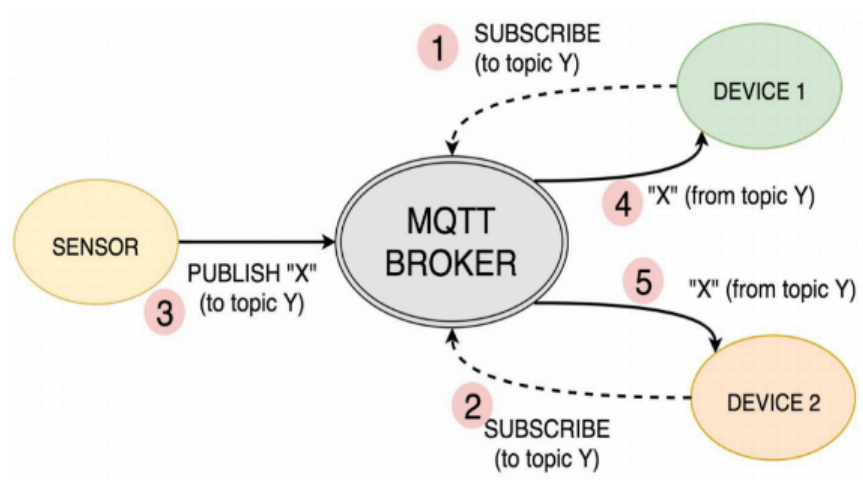

Figure 9. MQTT protocol

\section{Architecture of the Traffic Monitoring System}

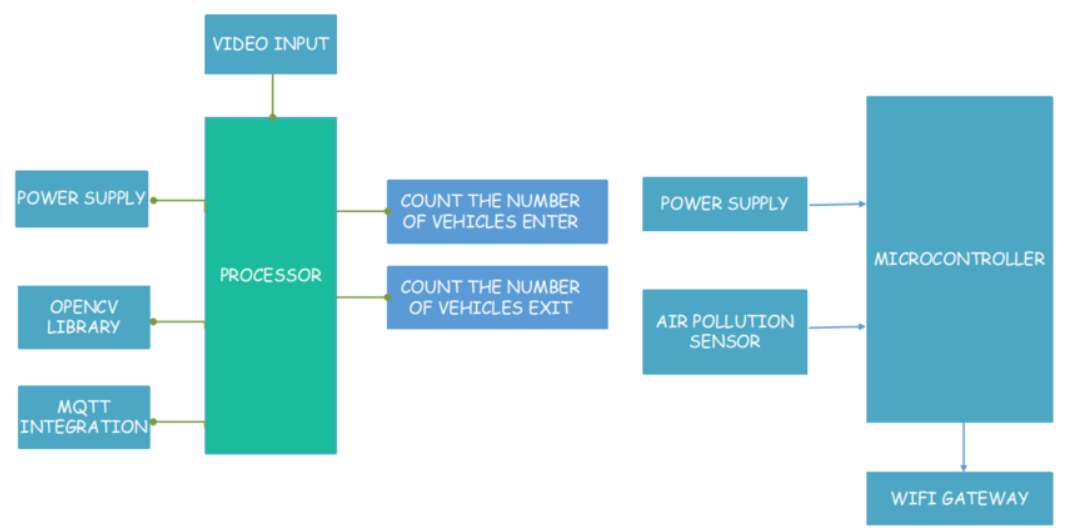

Figure 10. Architecture of the Traffic monitoring system 
A data set may be an assortment of information. Deep Learning has become the go-to technique for resolution several difficult real-world issues. Its positively out and away from the best-performing technique for pc vision tasks. The image on top of showcases the facility of deep learning for pc vision. With enough coaching, a deep network will phase and determine the key points of each person within the image. These deep learning machines that are operating therefore well want to fuel millions of fuel; that fuel is knowledge. And, within the deep learning era, knowledge is incredibly well arguably the foremost valuable resource. There square measure 3 steps of assembling knowledge

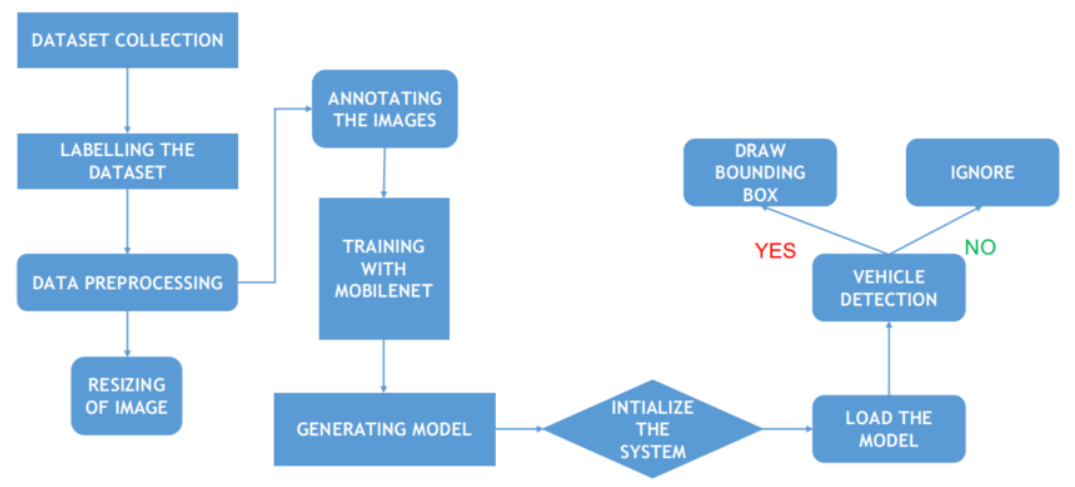

Figure 11. Atomobile Prediction algorithm

\section{Results}

The Figure 12 shows the count update after few seconds. The Figure 13 shows the complete hardware kit for chattelive air pollution. The Figure 14 shows traffic timing change after every 10 seconds. The Figure 15 shows the mitt mobile application for wireless

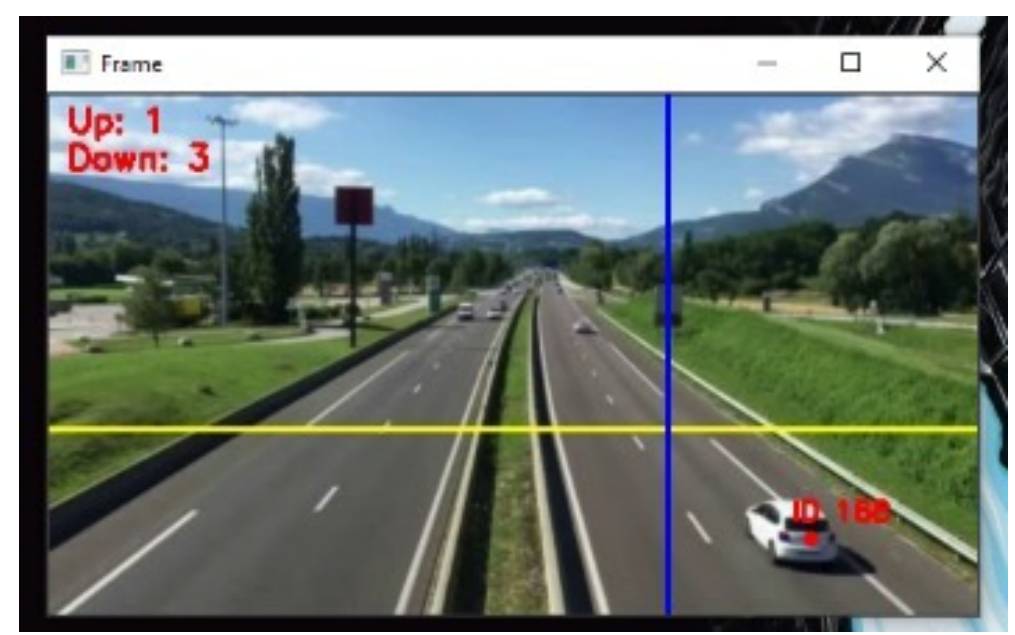

Figure 12. Count update after few seconds 
viewing of values. The Figure 16 shows graphical analysis of left and right count through the MQTT mobile application.

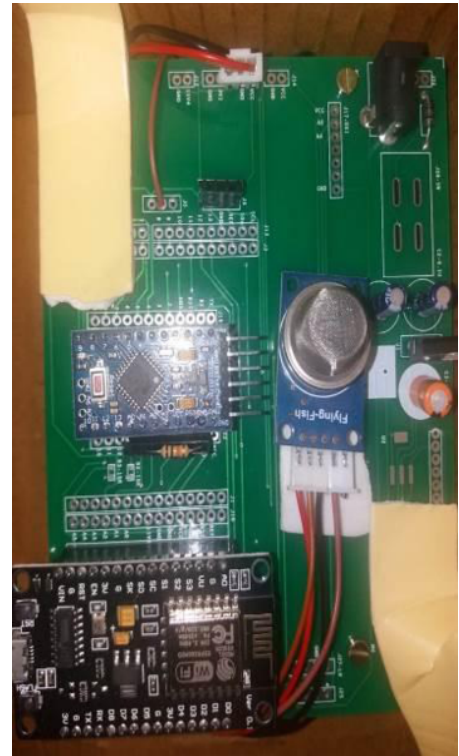

Figure 13. Hardware setup of the project

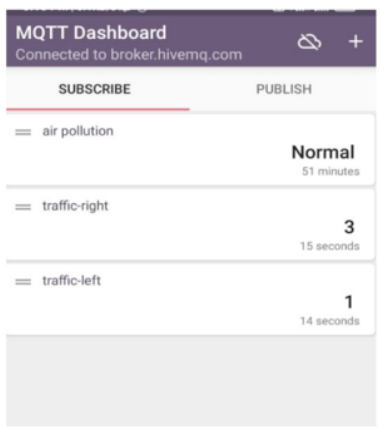

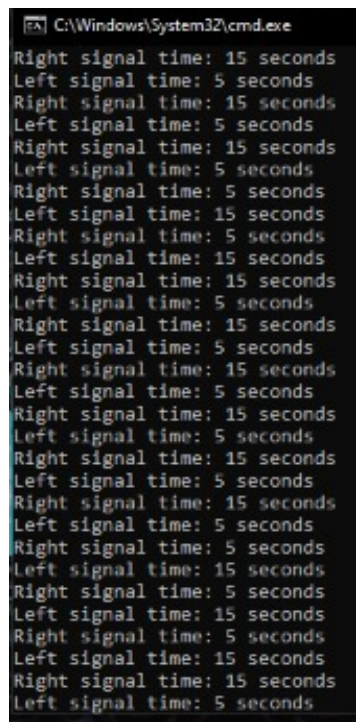

Figure 14. Traffic timing change after every 10 seconds

\begin{tabular}{|c|c|}
\hline $\begin{array}{l}\text { MQTT Dashb } \\
\text { Connected to bro }\end{array}$ & $\Delta+$ \\
\hline SUBSCRIBE & PUBLISH \\
\hline$=$ kcg_gas 1 & $\begin{array}{r}\text { Abnormal } \\
1 \text { second }\end{array}$ \\
\hline$=$ traffic-right & $\begin{array}{c}\mathbf{8} \\
1 \text { second }\end{array}$ \\
\hline$=$ traffic-left & $\underset{\text { now }}{9}$ \\
\hline
\end{tabular}

Figure 15. MQTT mobile app interface values
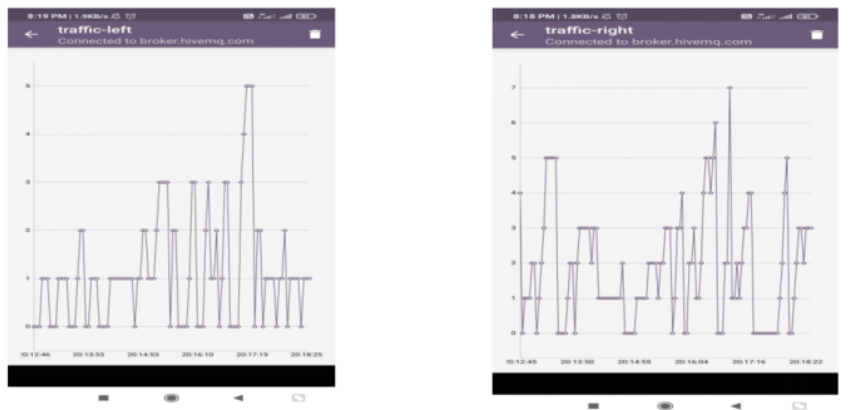

Figure 16. Graphical analysis of lest right count 


\section{Conclusion and future work}

This project is successfully implemented for effective traffic monitoring. This project is very helpful in reducing the burden on traffic management by the traffic department removing traffic congestion in certain areas. In the coming future, we review the application of the traffic monitoring technology in the traffic department and it can promote the advancement of the traffic management system with more accuracy. In this field, there is more chance to develop or convert this project in many ways. Thus, this project has an efficient scope in the coming future where this idea can be cheaply converted to computerized production.

\section{References}

[1] Prasath JS, Ramachandraiah U, Muthukumaran G. Modified Hardware Security Algorithms for Process Industries Using Internet of Things. Journal of Applied Security Research. 2021 Jan 2;16(1):127-40.

[2] Lin L, Li J, Chen F, Ye J, Huai J. Road traffic speed prediction: a probabilistic model fusing multi-source data. IEEE Transactions on Knowledge and Data Engineering. 2017 Jun 22;30(7):1310-23.

[3] Chen M, Yu X, Liu Y. PCNN: Deep convolutional networks for short-term traffic congestion prediction. IEEE Transactions on Intelligent Transportation Systems. 2018 Jun 21;19(11):3550-9.

[4] Hou Z, Li X. Repeatability and similarity of freeway traffic flow and long-term prediction under big data. IEEE Transactions on Intelligent Transportation Systems. 2016 Jan 26;17(6):1786-96.

[5] Backfrieder C, Ostermayer G, Mecklenbruker CF. Increased traffic flow through node-based bottleneck prediction and V2X communication. IEEE Transactions on Intelligent Transportation Systems. 2016 Jul 18;18(2):349-63.

[6] Singh D, Mohan CK. Deep spatio-temporal representation for detection of road accidents using stacked autoencoder. IEEE Transactions on Intelligent Transportation Systems. 2018 May 28;20(3):879-87.

[7] Anand J, Flora A. TG: Emergency traffic management for ambulance using wireless communication. IPASJ Int. J. Electron. Commun.(IIJEC). 2014;2(7):1-4.

[8] Payalan YF, Guvensan MA. Towards next-generation vehicles featuring the vehicle intelligence. IEEE Transactions on Intelligent Transportation Systems. 2019 Jun 11;21(1):30-47.

[9] Prasath JS, Jayakumar S, Karthikeyan K. Real-Time Implementation for Secure monitoring of Wastewater Treatment Plants using Internet of Things. International Journal of Innovative Technology and Exploring Engineering. 2019;9(1):2997-3002.

[10] Ahmed SA, Dogra DP, Kar S, Patnaik R, Lee SC, Choi H, Nam GP, Kim IJ. Query-based video synopsis for intelligent traffic monitoring applications. IEEE Transactions on Intelligent Transportation Systems. 2019 Jul 26;21(8):3457-68.

[11] Savino G, Pierini M, Baldanzini N. Decision logic of an active braking system for powered two wheelers. Proceedings of the Institution of Mechanical Engineers, Part D: Journal of automobile engineering. 2012 Aug;226(8):1026-36.

[12] Kaplan S, Guvensan MA, Yavuz AG, Karalurt Y. Driver behavior analysis for safe driving: A survey. IEEE Transactions on Intelligent Transportation Systems. 2015 Aug 26;16(6):3017-32.

[13] Zhao D, Huang X, Peng H, Lam H, LeBlanc DJ. Accelerated evaluation of automated vehicles in car-following maneuvers. IEEE Transactions on Intelligent Transportation Systems. 2017 May 25;19(3):733-44.

[14] Gillespie T, Hailes S. Assignment of Legal Responsibilities for Decisions by Autonomous Cars Using System Architectures. IEEE Transactions on Technology and Society. 2020 Aug 5;1(3):148-60.

[15] Luo Y. Environmental cost control of coal industry based on cloud computing and machine learning. Arabian Journal of Geosciences. 2021 Jun;14(12):1-6. 